\title{
Reduced fasting and postprandial triglyceride levels by alfrocumab in male diabetes patients on intensive insulin treatment by improved chylomicron metabolism
}

Benjamin Burggraaf ${ }^{1}$, Nadine Pouw ${ }^{2}$, Salvador Fernández Arroyo ${ }^{3}$, Leonie C. van Vark-van der Zee ${ }^{4}$ Gert-Jan M. van de Geijn², E. Birnie ${ }^{5}$, Jeannine Huisbrink ${ }^{6}$, Ellen M. van der Zwan², Monique T. Mulder ${ }^{4}$ Patrick C.N. Rensen7, Wouter W. de Herder ${ }^{4}$, Manuel Castro Cabezas ${ }^{1}$

Departments of ${ }^{1}$ Internal Medicine, ${ }^{2}$ Clinical Chemistry, ${ }^{5}$ Statistics and Education, 6 Pharmacy, Franciscus Gasthuis \& Vlietland, Rotterdam, the Netherlands Departament de 3 Medicina i Cirurgia, Unitat de Recerca Biomèdica, Universitat Rovira i Virgili, Tarragona, Spain

Department of ${ }^{4}$ Internal Medicine, Erasmus Medical Center, Rotterdam, the Netherlands

Department of 7 Internal Medicine, Division of Endocrinology, Leiden University Medical Center, Leiden, the Netherlands

\section{Introduction}

- Type 2 Diabetes Mellitus (T2DM) is associated with an increased risk for cardiovascular disease, atherogenic dyslipidemia and postprandial hyperlipidemia.

- Statins have shown to improve diabetic dyslipidemia.

- The effects of proprotein convertase subtilisin/kexin type 9 (PCSK9) inhibitors on postprandial lipemia in T2DM have not been evaluated in detail.

\section{Methods}

A randomized, 1:1, double-blind, proof-of-concept study in which 12 male patients with T2DM on intensive insulin regimen were allocated to 6 weeks of biweekly subcutaneous alirocumab (150 mg) or placebo. Postprandial lipemia was assessed before and after an intervention using an 8-hrs standardized oral fat loading test. Vascular function was assessed by measurement of fasting pulse wave velocity (PWV) and pulse wave augmentation index (Aix).

\section{Results}

Table 1. Baseline characteristics

\begin{tabular}{|l|c|c|c|} 
& $\begin{array}{c}\text { Total } \\
(\mathbf{n}=\mathbf{1 2})\end{array}$ & $\begin{array}{c}\text { Alirocumab } \\
(\mathbf{n}=\mathbf{6})\end{array}$ & $\begin{array}{c}\text { Placebo } \\
(\mathbf{n}=\mathbf{6})\end{array}$ \\
\hline Age (years) & $62.6 \pm 7.8$ & $61.5 \pm 9.9$ & $63.7 \pm 5.6$ \\
\hline Body mass index $\left(\mathrm{kg} / \mathrm{m}^{2}\right)$ & $32.8 \pm 5.6$ & $31.5 \pm 6.0$ & $34.1 \pm 5.5$ \\
\hline Waist circumference $(\mathrm{cm})$ & $110 \pm 21$ & $107 \pm 25$ & $113 \pm 19$ \\
\hline Systolic blood pressure $(\mathrm{mmHg})$ & $134 \pm 11$ & $133 \pm 12$ & $135 \pm 10$ \\
\hline Fasting glucose $(\mathrm{mmol} / \mathrm{L})$ & $8.5 \pm 1.9$ & $8.3 \pm 2.3$ & $8.6 \pm 1.7$ \\
\hline Glycated A1c $(\mathrm{mmol} / \mathrm{mol})$ & $65.6 \pm 8.7$ & $63.5 \pm 7.7$ & $65.5 \pm 10.9$ \\
\hline LDL-C (mmol/L) & $1.9 \pm 0.5$ & $1.9 \pm 0.5$ & $2.0 \pm 0.5$ \\
\hline Plasma TG $(\mathrm{mmol} / \mathrm{L})$ & $1.89(0.92-2.87)$ & $1.75(0.86-2.59)$ & $2.64(1.69-3.59)$ \\
\hline Apo B (g/L) & $0.85 \pm 0.20$ & $0.82 \pm 0.15$ & $0.89 \pm 0.25$ \\
\hline Apo B48 (mg/L) & $12.9(4.4-21.4)$ & $9.3(0.0-19.2)$ & $18.8(8.2-29.4)$ \\
\hline \multicolumn{2}{|c|}{ Abbreviations: Apo, apolipoprotein; LDL-C, low-density lipoprotein cholesterol; TG, triglycerides }
\end{tabular}

Fasting levels of total cholesterol (median change compared to baseline $-28.2 \% ; \mathrm{p}<0.001)$, LDL-C $(-39.5 \% ; \mathrm{p}<0.001)$ and apo B $(-50.7 \% ; \mathrm{p}<0.001)$ were significantly changed after six weeks of treatment with alirocumab versus placebo.

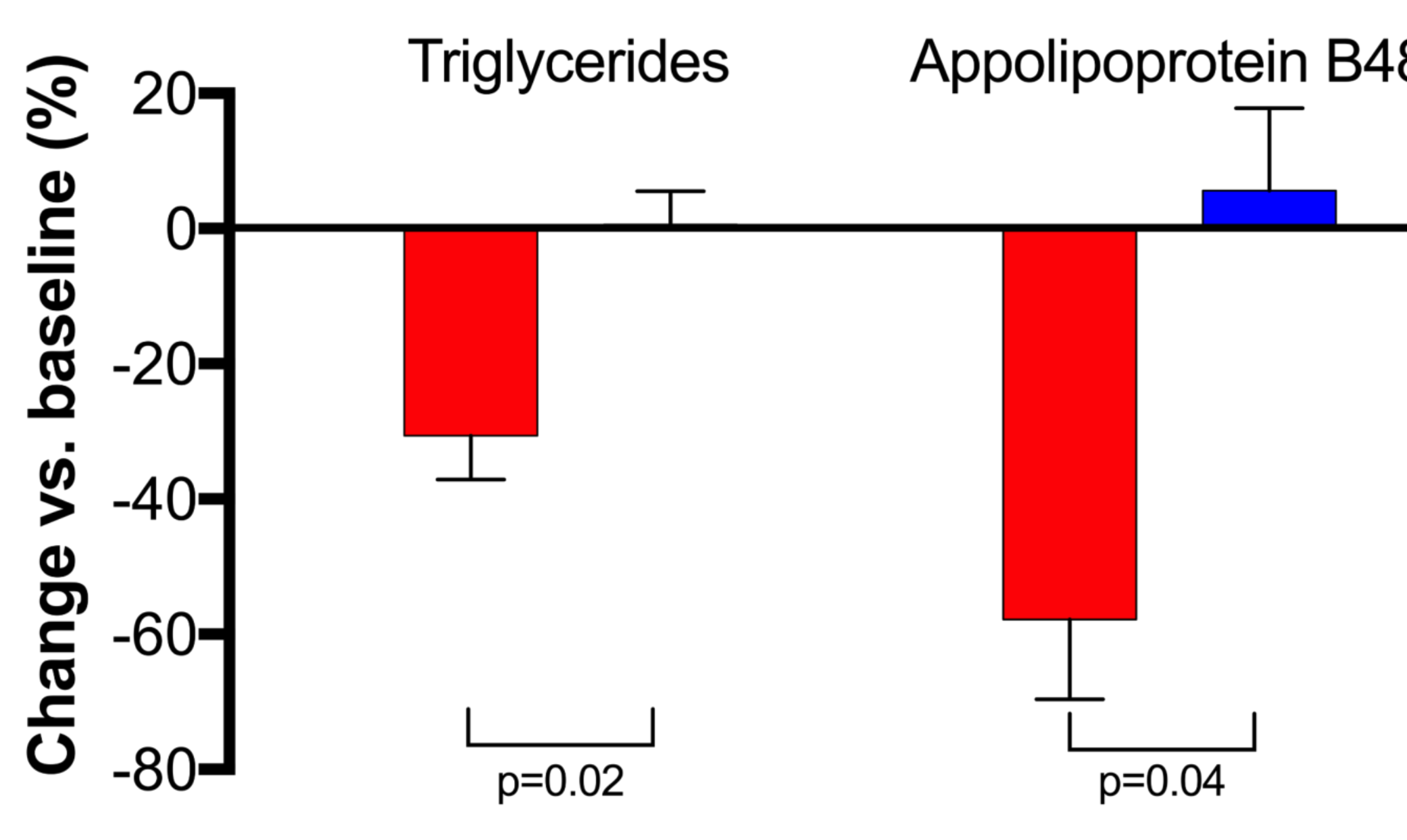

A median $-24.7 \%$ $(p=0.02)$ change compared to baseline in fasting triglycerides and a median $-35.9 \%$ $(p=0.04)$ change in apo B48 levels compared to baseline was observed between alirocumab (red bar) and placebo (blue bar).
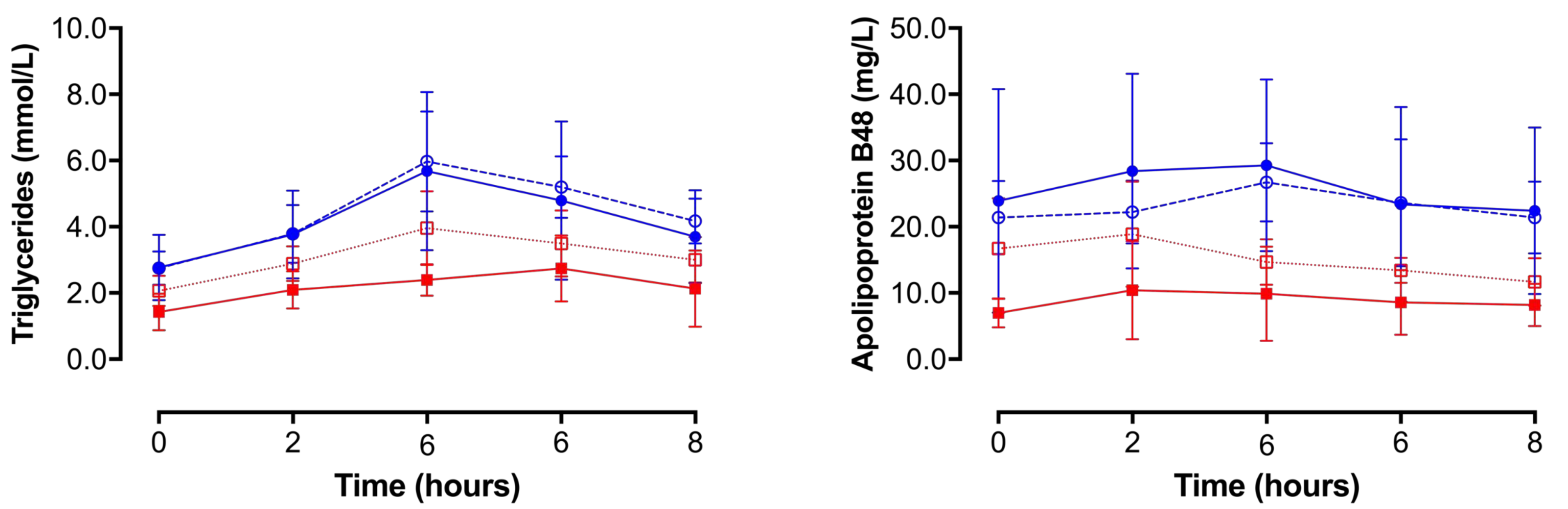

Figure 1. Mean (standard error of the mean) of TG and apo B48 levels during an oral fat load with alirocumab at baseline (red open square) and after treatment (red closed square) and placebo at baseline (blue open circle) and after treatment (blue closed circle).

Postprandial (incremental area under the curve) responses of total cholesterol (median change compared to baseline $-28.2 \%$; $p<0.001)$, apolipoprotein B $(-48.6 \%$; $\mathrm{p}<0.001)$ and HDL-C $(+4.2 \% ; \mathrm{p}=0.04)$ changed after six weeks of treatment with alirocumab compared to placebo.

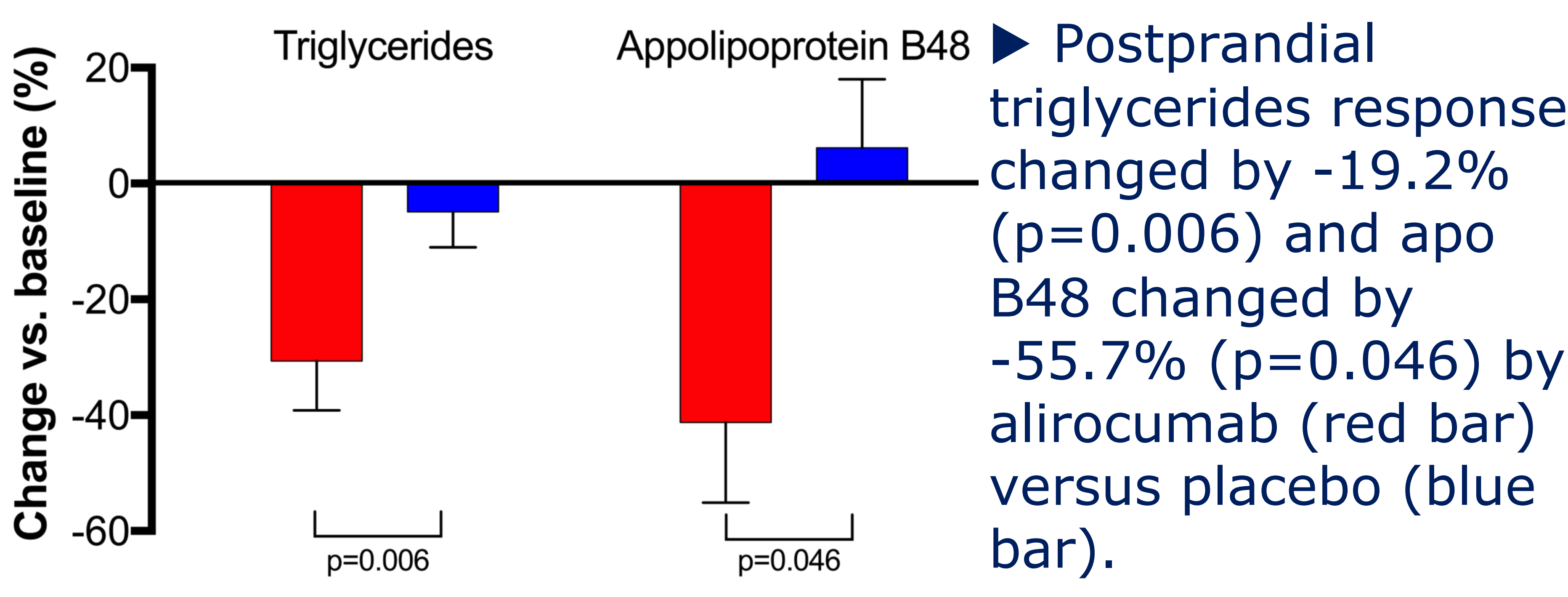

Fasting pulse wave velocity was reduced after six weeks of alirocumab versus placebo (median change compared to baseline $-7.9 \% ; p=0.03)$. There were no changes in postprandial vascular function (both PWV and Aix).

\section{Conclusion}

Alirocumab lowered both fasting and postprandial TG levels in male patients with T2DM by reducing levels of circulating chylomicrons and their remnants. In addition, an improved vascular elasticity was found.

This may contribute to cardiovascular protection in T2DM in addition to LDL-C lowering by these compounds. 